\title{
Chimpanzees, cooking, and a more comparative psychology
}

\author{
Michael J. Beran ${ }^{1} \cdot$ Lydia M. Hopper $^{2}$ • Frans B. M. de Waal ${ }^{3} \cdot$ Sarah F. Brosnan $^{1}$ • \\ Ken Sayers ${ }^{1}$
}

Published online: 11 April 2016

(C) Psychonomic Society, Inc. 2016

\begin{abstract}
A recent report suggested that chimpanzees demonstrate the cognitive capacities necessary to understand cooking (Warneken \& Rosati, 2015). We offered alternative explanations and mechanisms that could account for the behavioral responses of those chimpanzees, and questioned the manner in which the data were used to examine human evolution (Beran, Hopper, de Waal, Sayers, \& Brosnan, 2015). Two commentaries suggested either that we were overly critical of the original report's claims and methodology (Rosati \& Warneken, 2016), or that, contrary to our statements, early biological thinkers contributed little to questions concerning the evolutionary importance of cooking (Wrangham, 2016). In addition, both commentaries took issue with our treatment of chimpanzee referential models in human evolutionary studies. Our response offers points of continued disagreement as well as points of conciliation. We view Warneken and Rosati's general conclusions as a case of affirming the consequent-a logical conundrum in which, in this case, a demonstration of a partial list of the underlying abilities required for a cognitive trait/suite (understanding of cooking) are suggested as evidence for that ability. And although we strongly concur with both Warneken and Rosati (2015) and Wrangham (2016) that
\end{abstract}

Michael J. Beran

mjberan@yahoo.com

1 Department of Psychology and Language Research Center, Georgia State University, University Plaza, Atlanta, GA 30302, USA

2 Lincoln Park Zoo, Chicago, IL, USA

3 Emory University, Atlanta, GA, USA chimpanzee research is invaluable and essential to understanding humanness, it can only achieve its potential via the holistic inclusion of all available evidence - including that from other animals, evolutionary theory, and the fossil and archaeological records.

Keywords Chimpanzees $\cdot$ Cooking $\cdot$ Evolution $\cdot$ Hominids Learning $\cdot$ Cognition

Rosati and Warneken (2016) and Wrangham (2016) have produced thoughtful responses to our review that can do much to further the conversation about the ideals of comparative psychology, as well as the requisite capacities for cooking. We believe that the individuals involved in this debate are, as a whole, fully committed to the idea that there is tremendous value in research assessing the behavior and cognition of chimpanzees, other great apes, and other primate species. It was not our intention to argue that one should not work with chimpanzees, or to deny that such work can contribute to a better understanding of human cognition (or, more broadly, of cognition itself, independent of species). All of the authors on the review article and the commentaries, including ourselves, rely heavily on chimpanzee research to contribute ideas to psychology, biology, and evolutionary theory, and we fully agree that this species is both important for this research agenda and interesting in its own right. We owe a debt of gratitude to chimpanzees for what we have learned through their involvement in psychological research. Here there is likely a broad consensus, even if opinions vary on just how good a model the chimpanzee is for human evolution, or whether the behavioral responses of chimpanzees to specific experimental tests can be used to infer higher-order cognitive capacities in specific cases. 


\section{Methodology reconsidered}

In their commentary, Rosati and Warneken (2016, p. 2) state the following about their original article (Warneken \& Rosati, 2015):

Based on this whole sequence of experiments, we argued that chimpanzees show several of the psychological capacities that are necessary to cook food: motivation or desire to pursue cooked food; patience to wait temporal delays to acquire cooked foods as well as pay some additional energetic cost to transport food so it would be cooked; the self-control to give up food in one's hand to have it be cooked; a basic causal comprehension of how these devices transformed raw foods after little experience; and finally the ability to save raw food for future cooking opportunities.

We agree that all of the points above were demonstrated, except one. We continue to disagree that the chimpanzees needed a "basic causal comprehension of how these devices transformed raw foods after little experience." It remains as plausible that the chimpanzees associated one stimulus (the cooking device) with high preference foods, and were attracted to it (whether choosing it, putting things into it, etc.), without needing an understanding of the transformation process. As we noted in our original review, there is no clear reason to prefer a causal-understanding interpretation over an associatively learned explanation. This position does not diminish the "smart-looking" choices of these chimpanzees, it simply means that we remain agnostic as to the mechanism (or mechanisms) underlying their behaviors. The chimpanzees may fully understand that this device "cooked" food (in whatever way they might think about cooking), but these data in total do not more strongly support that conclusion than one grounded in more basic forms of learning, at least without some evidence of the capacity to create or innovate a novel technique to transform the food.

Rosati and Warneken (2016, p. 3) also state that "To our knowledge, there is no overarching theory that specifies the necessary and sufficient behavioral skills for organisms to cook. Therefore, our approach was to suggest and test for basic cognitive and behavioral skills whose absence would preclude cooking behaviors." Cooking can be objectively defined as requiring some suite of behavioral and cognitive capacities, and some definitions do exist (e.g., Short, 2003, outlined the mechanical skills, perceptual abilities in terms of taste, color and texture of food, and knowledge of physics required to cook). We agree that chimpanzees demonstrate some of those capacities, but we do not agree that such premises necessarily lead to the conclusion that chimpanzees understand or appreciate cooking as a process that transforms the same item of food from one state (uncooked) to another (cooked), even if they can learn to obtain cooked foods through their behavioral responses.

In the present case, the argument goes like this: Take a behavior (such as cooking), break it into constituent pieces that are all necessary, and then use evidence of those components to argue that there is a capacity for that behavior (such as for cooking). The problem is that this is the logical error of affirming the consequent. Having some, or even all, of the requisite capacities that are part of a larger behavioral capacity or concept does not tell us anything about the necessary capability for that behavior or for knowledge of that concept. This argument only becomes invalidated when one finds a species that cannot exhibit self-control or planning, thereby necessarily being excluded from being capable of cooking (a point raised by Rosati \& Warneken, 2016). Indeed, Rosati and Warneken acknowledged that simply showing constituent abilities that, when combined, could generate a complex behavior does not mean those constituent abilities would generate a complex behavior. Beyond which, we note (as do Rosati \& Warneken, 2016) that it is unlikely that Warneken and Rosati (2015) identified all, or even the most important, factors that precipitated fire use and cooking in early hominids: As we noted in our original review, an unusual (even by primate standards) predilection toward information-seeking and curiosity, and exceptional episodic memory capacities, would also undoubtedly rate high on this list, as would factors such as food competition, tolerance, and cooperation, which would be necessary to delay the immediate consumption of captured food in wild chimpanzees.

A second point of disagreement between us and Warneken and Rosati (2015) comes from statements such as that chimpanzees have "a practical understanding of this basic cooking transformation after minimal experience." Understanding a process - or even a "practical understanding," which we assume means in a "folk psychology" kind of way - and learning about contingencies for behaviors are not the same thing. There can be very different interpretations of observed behaviors, and the burden is on experimenters to distinguish between them. This is not a criticism of the design of the original study or the effort given to execute it - which we know was monumental, given how hard it is to do this kind of research with chimpanzees - but a difference of opinion on the interpretation of the data.

We have outlined why eating high-preference foods, and why taking more familiar and low-preference foods to the cooker over taking unfamiliar things, are both explained by competing response strengths (Beran, Hopper, de Waal, Sayers, \& Brosnan, 2015). Rosati and Warneken (2016) suggest that the speed with which the chimpanzees learned to use the "cooking pot" to get preferred cooked food might argue against an associative-learning interpretation, and this is a 
reasonable claim. However, we do not know how many trials chimpanzees need to learn such new associations or whether other species could pass these tests just as quickly. We are inclined to accept that chimpanzees can generalize what they understand about test apparatuses rather quickly. But that does not mean that such flexible and objectively "smart" behavior must rely at all on understanding the idea of cooking (in whatever form) versus knowing how to get the best thing possible and then structuring behavior to get that. Note that there is no disagreement about self-control or planning abilities; the issue is whether those demonstrations were sufficient for claiming an understanding of cooking behavior. Indeed, Rosati and Warneken argue that although each of our individual points is true, we lacked an overarching theory to explain their entire suite of data, a condition met by cooking. We hope we have made clear that we think that the best overall explanation for the (impressive) suite of skills demonstrated in the original study is the chimpanzees' intelligent, flexible, and rapid learning, but not necessarily a "practical understanding of cooking" per se. The domain-general cognitive capacities required for cooking proposed by Warneken and Rosati (2015) could collectively allow for cooking - as they also would for many other behaviors (e.g., hunting and foraging) - but as we discussed above, simply demonstrating evidence for each (even collectively) does not allow for the conclusion that control of fire is the only missing element from chimpanzees cooking.

In tests of self-control, it is well-established that when presented with two options, whether quantitatively or qualitatively different, chimpanzees will point to the best thing (e.g., Boysen \& Berntson, 1995). In the test given to chimpanzees by Warneken and Rosati (2015), the choice of the larger amount (three items over one item) was also what was called the self-controlled response. There is no way to distinguish in that choice whether the chimpanzees were choosing to wait, or wanted more food with no aspect of delay factoring into that choice. However, Warneken and Rosati reported a difference between one raw versus three raw items and one raw versus three cooked items. The reverse-reward paradigm (Boysen \& Berntson, 1995) has consistently shown that the greater the discrepancy between options, the worse the subject performs. Given this, it would have been surprising to see anything other than the given outcome. We agree that the chimpanzees would more often point to three cooked pieces versus one raw piece than they would point to three raw pieces versus one raw piece, but we do not see this as necessarily reflecting anything about the degree of self-control they exhibited as a function of what they could obtain. Finally, we leave it to the reader to decide whether Clever Hans effects should no longer be considered a concern in comparative methodology (see Beran, 2012). We argue that it is important to control for possible inadvertent cues given to subjects, even if the use of such cues is not expected to occur in some tests or with some species.
The origins of cooking: Historical considerations

In our review, we cited Darwin (1874/1998) and Engels $(1876 / 1953)$ as among those that have recognized the evolutionary importance of cooking, and later clarified their specific involvement: recognizing the value of detoxifying and/or digesting foods while it was still outside the body. Wrangham (2016) provides relevant historical quotes but does not accept that these authors were dealing with "evolutionary effects." Wrangham and his colleagues (e.g., Wrangham 2007, 2009; Wrangham \& Conklin-Brittain, 2003; Wrangham et al., 1999) have considered cooking and its potential behavioral and anatomical consequences more thoroughly, by far, than any other authors. Our goal was simply to give appropriate credit to these earlier thinkers who discussed cooking in works explicitly devoted to human evolution, and noted the primary functional importance of this behavior. Indeed, their functional explanationthe processing of edibles before consumption-is central to many of the morphological and other cooking-related changes proposed by Wrangham and other authors.

\section{Chimpanzee models for human evolution}

Both commentaries (Rosati \& Warneken, 2016; Wrangham, 2016) are concerned over our discussion on the role of chimpanzees in human evolutionary studies. We concur that chimpanzee data should play a crucial role in this work, and did not intend to imply otherwise. Make no mistake: Pan troglodytes has been, is, and will continue to be an important source of information for this field of research. Our point was simply that this species is not the only source of information, and attempting to project the characteristics of a singular living terminal taxon to creatures separated from it by eight or more million years of evolution comes with issues that must be addressed - particularly when it comes to complex behavioral-cognitive suites, such as those related to the advent of cooking. Because we cannot, unfortunately, study earlier members of the human family tree, it is particularly important to utilize a wide taxonomic range in drawing conclusions about human evolution.

It is true that chimpanzees have brains only marginally smaller than australopithecines, and many scholars consider chimpanzee cognitive abilities to be a convenient shorthand for the minimum found in the Pan-Homo last common ancestor, or even early hominids (for endocast evidence of neural reorganization in Australopithecus, differing from extant apes, see Carlson et al., 2011; Dart, 1925; Falk, 2009; Holloway, Clarke, \& Tobias, 2004). On the face of it, nothing would seem particularly controversial about this (but see Sayers \& Lovejoy, 2008). Phylogenetics, however, is by nature a comparative enterprise - and the more comparative it is, 
the better. Indeed, in the most detailed application of formal phylogenetic methods hitherto utilized to reconstruct the Pan-Homo common ancestor-which drew widely from a large number of catarrhine primates - the results were so divergent from the chimpanzee that the authors concluded that utilizing "single extant species to explain behavioral evolution of early hominins is ... of limited use" (Duda \& Zrzavý, 2013, p. 424).

Utilizing data from myriad species is vastly superior to limiting our studies to chimpanzees (or any other species, for that matter) and results in firmer conclusions. What would it mean, for example, if capuchin monkeys or lemurs performed equivalently to the chimpanzees in the Warneken and Rosati (2015) study? Would this dampen claims that these behavioral suites were tied to eventual control of cooking by humans? As we noted in our original article, relevant, published data from other primates, and from nonprimates such as rats, shed light on all of the listed "prerequisite abilities" for a concept of cooking. If you want to truly understand the origins and evolution of self-control, or planning, or episodic memory, or any other psychological feature, the taxonomic net must be cast widely. Of course, we recognize that in any single study we cannot possibly hope to cast the taxonomic net as widely as we are recommending; that is a career's endeavor. Nonetheless, it is important not to implicitly assume that the data of any one species are either sufficient or superior to the broader comparative view, and to incorporate available evidence from other species when drawing conclusions from data. All relevant information - the fossil and archaeological records, rules of ecology or behavior derived from studies of many species, modern phylogenetic methods, and so onmust be brought to bear on the issues at hand. Chimpanzees will continue to be an integral part of this work, but not the only part. This broader comparative approach is the most likely route toward genuine insights concerning human origins.

Author Note All authors contributed to writing this article.

\section{References}

Beran, M. J. (2012). Did you ever hear the one about the horse that could count? Frontiers in Psychology, 3, 357. doi:10.3389/fpsyg.2012. 00357

Beran, M. J., Hopper, L. M., de Waal, F. B. M., Sayers, K., \& Brosnan, S. F. (2015). Chimpanzee food preferences, associative learning, and the origins of cooking. Learning \& Behavior. Advance online publication. doi:10.3758/s13420-015-0206-x

Boysen, S. T., \& Berntson, G. G. (1995). Responses to quantity: Perceptual versus cognitive mechanisms in chimpanzees (Pan troglodytes). Journal of Experimental Psychology: Animal Behavior Processes, 21, 82-86. doi:10.1037/0097-7403.21.1.82

Carlson, K. J., Stout, D., Jashashvili, T., de Ruiter, D. J., Tafforeau, P., Carlson, K., \& Berger, L. R. (2011). The endocast of MH1, Australopithecus sediba. Science, 333, 1402-1407. doi:10.1126/ science. 1203922

Dart, R. A. (1925). Australopithecus africanus: The man-ape of South Africa. Nature, 115, 195-199.

Darwin, C. (1998). The descent of man, and selection in relation to sex (2nd ed.). Amherst, NY: Prometheus (Original work published 1874).

Duda, P., \& Zrzavý, J. (2013). Evolution of life history and behavior in Hominidae: Towards phylogenetic reconstruction of the chimpanzee-human last common ancestor. Journal of Human Evolution, 65, 424-446. doi:10.1016/j.jhevol.2013.07.009

Engels, F. (1953). The part played by labour in the transition from ape to man. Moscow, Russia: Foreign Languages Publishing House (Original work published 1876).

Falk, D. (2009). The natural endocast of Taung (Australopithecus africanus): Insights from the unpublished papers of Raymond Arthur Dart. Yearbook of Physical Anthropology, 52(Suppl. 49), 49-65.

Holloway, R. L., Clarke, R. J., \& Tobias, P. V. (2004). Posterior lunate sulcus in Australopithecus africanus: Was Dart right? Comptes Rendus Palevol, 3, 287-293.

Rosati, A. G., \& Warneken, F. (2016). How comparative psychology can shed light on human evolution: Response to Beran et al.'s discussion of "Cognitive capacities for cooking in chimpanzees." Learning \& Behavior. Advance online publication. doi:10.3758/ s13420-016-0220-7

Sayers, K., \& Lovejoy, C. O. (2008). The chimpanzee has no clothes: A critical examination of Pan troglodytes in models of human evolution. Current Anthropology, 49, 87-114. doi:10.1086/523675

Short, F. (2003). Domestic cooking skills-What are they? Journal of the HEIA, 10, 13-22.

Warneken, F., \& Rosati, A. G. (2015). Cognitive capacities for cooking in chimpanzees. Proceedings of the Royal Society B, 282, 20150229. doi:10.1098/rspb.2015.0229

Wrangham, R. W. (2007). The cooking enigma. In C. Pasternak (Ed.), What makes us human? (pp. 182-203). Oxford, UK: Oneworld.

Wrangham, R. W. (2009). Catching fire: How cooking made us human. New York, NY: Basic Books.

Wrangham, R. W. (2016). The curiously long absence of cooking in evolutionary thought. Learning \& Behavior. Advance online publication. doi:10.3758/s13420-016-0223-4

Wrangham, R. W., \& Conklin-Brittain, N. L. (2003). Cooking as a biological trait. Comparative Biochemistry and Physiology Part A, 136, 35-46. doi:10.1016/S1095-6433(03)00020-5

Wrangham, R. W., Jones, J. H., Laden, G., Pilbeam, D., \& ConklinBrittain, N. L. (1999). The raw and the stolen: Cooking and the ecology of human origins. Current Anthropology, 40, 567-594. doi:10.1086/300083 